\title{
ELECTRIC MANIFESTATIONS AND SOCIAL IMPLICATIONS OF BACTERIA AGGREGATION FROM THE BESSEL-KELLER-SEGEL EQUATION
}

\author{
Huber Nieto-Chaupis \\ Universidad Autónoma del Perú \\ Panamericana Sur Km. 16.3 Villa el Salvador, Lima PERÚ \\ NOTE: Corresponding author: hubernietochaupis@gmail.com
}

\begin{abstract}
This paper proposes a fair extension of Keller-Segel equation based in the argument that bacteria exhibit proporties electric in their composition. The new mathematical form of this extension involves the integer-order Bessel functions. With this one can go through the electrodynamics of the representative scenarios in order to understand the social behavior of bacteria. From the theoretical side this paper demonstrates that, charged electrically, aggregation of bacteria would give rise to electric currents that hypothetically are the reasons for social organization and disruption among them. The electrical properties of bacteria from this mathematical proposal might be relevant in a prospective implementation of so-called Internet of Bio-Nano Things network, that aims to be characterized for having a very high signal/noise.
\end{abstract}

Keywords - Bessel, classical electrodynamics, Keller-Segel, nanonetwork

\section{INTRODUCTION}

Evelyn Keller and Lee Segel in 1971 reported in [1] that bacteria exhibit some relative preference to move through the highest concentrations of substrate instead of the lowest ones. This phenomenon was projected onto the equation known as the Keller-Segel equation and it is written as:

$$
\frac{\partial b(x, t)}{\partial t}=\frac{d}{d x}\left[\mu(s) \frac{d b(x, t)}{d x}\right]-\frac{d}{d x}\left[b(x, t) \chi(s) \frac{d s}{d x}\right]
$$

whose meaning of elements of this reads as follows:

- $\boldsymbol{b}(\boldsymbol{x}, \boldsymbol{t})=$ bacteria density,

- $\boldsymbol{s}(\boldsymbol{x}, \boldsymbol{t})=$ substrate density,

- $\chi(s)=$ chemotactic coefficient,

- $\boldsymbol{\mu}(\boldsymbol{s})=$ motility parameter.

Eq.1 can be seen actually as a kind of compensation. In effect, one can see that by putting $\chi(s, t)$ and $\boldsymbol{\mu}(\boldsymbol{s})$ as constants, then the positive change $\frac{\partial \boldsymbol{b}(x, t)}{\partial \boldsymbol{t}}>0$ demands that:

$$
\frac{d^{2} b(x, t)}{d x^{2}}>\frac{d^{2} s(x, t)}{d x^{2}} .
$$

That is valid over the allowed periods of bacteria substrate interaction. This has interesting consequences in the discipline of bacteria dynamics [2]. Clearly one can see that it encompasses the reason of the Keller-Segel equation, in the sense that bacteria can deplete substrate in an efficient manner. For example, Unluturk, Balasubramanian and Akyildiz [3] have used the Keller-Segel model in the study of social behavior of bacteria inside the framework of molecular communications as part of the fundamental postulates of the prospective Internet of Bio-Nano Things (IoBNT) [4]. Clearly one can appeal to physics of Eq. 1 for a robust application inside the molecular and phenomena at the nano-level. Since bacteria is transporting a net electric charge, then a plethora of ways to search their behavior represents an option to use theoretical scenarios of physics interactions and the implcations that would rise in a scenario of bionano technology. Thus, Eq. 1 opens various paths to understand both the physics and biophysics of bacteria dynamics. Of course, Eq. 1 can also be seen as a fair extension of diffusion equation [5]. In effect, Eq.1 to some extent can also be written as $\frac{\partial \rho(s, t)}{\partial t}=\mathrm{D} \frac{\partial^{2} \rho(s, t)}{\partial s^{2}}+\mathrm{G}(\mathrm{s}, \mathrm{t})$ with $\mathrm{D}$ the diffusion constant and $G(s, t)$ enclosing a set of operations based at both spatial and time derivatives. Thus, emerges the questions: Are bacteria fully diffusive? What are then the physics of this possible diffusion? Here one can answer in terms of physics laws in: (i) Electricity, (ii) Thermodynamics, and (iii) Space-time propagation. The purpose of this paper is to extend Eq.1 when bacteria are composed by electrical material such as ions for instance, so that concrete electric interactions would take place. 
In this manner a large amount of bacteria density might be described by micro-forces so that a combined description based in Newton forces and electrodynamics can add relevant information to the process of substrate degradation. In cases of complex dynamics, of course the usage of diffusion equation can be obsolete so that an upgraded version would have to be needed. For example, one can see the work of Rosen in 1984 [6] characterized by having differential equations inspired by the Navier-Stokes scenario in order to propose a theory of bacteria chemotaxis. The contribution of this paper is in essence the reformulation of the KellerSegel equation containing the integer-order Bessel functions. With this an analysis about the implications of electrodynamics is derived. The rest of this paper is structured as follows: In the second section, the Bessel-Keller-Segel equation is derived. Once this equation is established then in the third section the eletrodynamics equations are derived in a closed-form manner. Here the instantaneous electric current is presented. It is seen that the resulting distributions exhibit a certain similarity with the discharge of a typical RC circuit. Finally, in fourth section the conclusion of the paper is presented.

\section{EXTENSION OF THE KELLER-SEGEL EQUATION}

Actually, one can exploit Eq. 1 in many ways by which one can extract the dynamics of any aggregation of bacteria in different scenarios while under interaction with substrates [7], [8]. Bacteria as a biological unit can require particular capabilities in order to guarantee an optimal colonization. Although it is not clear whether bacteria dynamics behave as a linear or nonlinear system, throughtout this document it will be assumed the linear assumption that allows for exact extensions.

Thus, for instance consider the case where $\boldsymbol{\mu}(\boldsymbol{s})=$ $\boldsymbol{g}(\boldsymbol{s}) \equiv \boldsymbol{g}(\boldsymbol{x})$ and $\chi(\boldsymbol{s})=\boldsymbol{h}(\boldsymbol{s}) \equiv \boldsymbol{h}(\boldsymbol{x})$ acquiring an explicit dependence on " $\boldsymbol{x}$ " then from Eq. 1 one arrives at:

$$
\begin{gathered}
\frac{\partial b(x, t)}{\partial t}=g(x)\left[\frac{d^{2} b(x, t)}{d x^{2}}\right]-h(s)\left[\frac{d b(x, t)}{d x} \frac{d s}{d x}\right] \\
-h(s)\left[b(x, t) \frac{d^{2} s}{d x^{2}}\right]
\end{gathered}
$$

One interesting scenario is the stationary one with $\frac{\partial \boldsymbol{b}(\boldsymbol{x}, \boldsymbol{t})}{\boldsymbol{\partial t}}=\mathbf{0}$. In this manner one can assume the following: $\boldsymbol{h}(\boldsymbol{x}) \boldsymbol{d s} / \boldsymbol{d x}=-\boldsymbol{x}$ in the second term in the right side of Eq. 3, yielding:

$$
\frac{d s(x, t)}{d x}=\frac{-x}{h(s)} \Rightarrow s(x, t)=-\int \frac{x}{h(s)} d x .
$$

In this manner the Keller-Segel equation with $\boldsymbol{g}(\boldsymbol{x})=\boldsymbol{x}^{2}$ one gets:

$x^{2}\left[\frac{d^{2} b(x, t)}{d x^{2}}\right]+x\left[\frac{d b(x, t)}{d x}\right]-h(x)\left[b(x, t) \frac{d^{2} s}{d x^{2}}\right]=0$.

\subsection{Essential Assumptions}

Eq.5 lends itself to being operated towards two known differential equations. Although one can apply unphysical procedures, it is clear that in a first instance the Bessel structure can be a good candidate. In this manner, in order to construct an integer-order Bessel differential equation [9], one needs to impose at the rigth-side the following:

$$
h(s) \frac{d^{2} s}{d x^{2}}=-\left(1-\frac{n^{2}}{x^{2}}\right)
$$

that implies that there is a direct relation between the function $\boldsymbol{h}(\boldsymbol{s})$ and the integer numbers $\boldsymbol{n}$. On the other hand one can take the definition of Eq. 4 to be inserted in Eq. 6 yielding:

$$
-h(x) \frac{d}{d x}\left[\frac{x}{h(s)}\right]=\left(1-\frac{n^{2}}{x^{2}}\right)
$$

where the signs have been canceled in both sides. Subsequently by applying the derivative in a straightforward manner then Eq. 7 can be read as:

$$
\begin{gathered}
-h(x)\left[\frac{h(s)-x \frac{d h}{d s} \frac{d s}{d x}}{h^{2}(s)}\right]= \\
-\left[\frac{h(s)-x \frac{d h}{d s} \frac{d s}{d x}}{h(s)}\right]=\left(1-\frac{n^{2}}{x^{2}}\right)
\end{gathered}
$$

yielding a first-order differential equation that can be written as:

$$
\Rightarrow x \frac{d h}{d s}-h(s)=h(s)\left(1-\frac{n^{2}}{x^{2}}\right) .
$$

It was assumed the approximation given by $\frac{d s}{d x} \approx 1$, (resulting in a toy model for substrate dynamics that appears to be linear with $x$ ). It allows a direct solution of first-order differential equation that can be written as:

$$
\frac{d h}{h}=\left(\frac{2}{x}-\frac{n^{2}}{x^{3}}\right) d s \quad \Rightarrow \quad h(s)=\operatorname{Exp}\left[s\left(\frac{2}{x}-\frac{n^{2}}{x^{3}}\right)\right] .
$$


In this manner the chemotactic coefficient is expressed in terms of " $x$ ". A direct solution for it yields the exponential form and depends directly on the substrate density " $s$ ". Therefore, one arrives at:

$$
\Rightarrow \chi(s)=\operatorname{Exp}\left[s(x)\left(\frac{2}{x}-\frac{n^{2}}{x^{3}}\right)\right]
$$

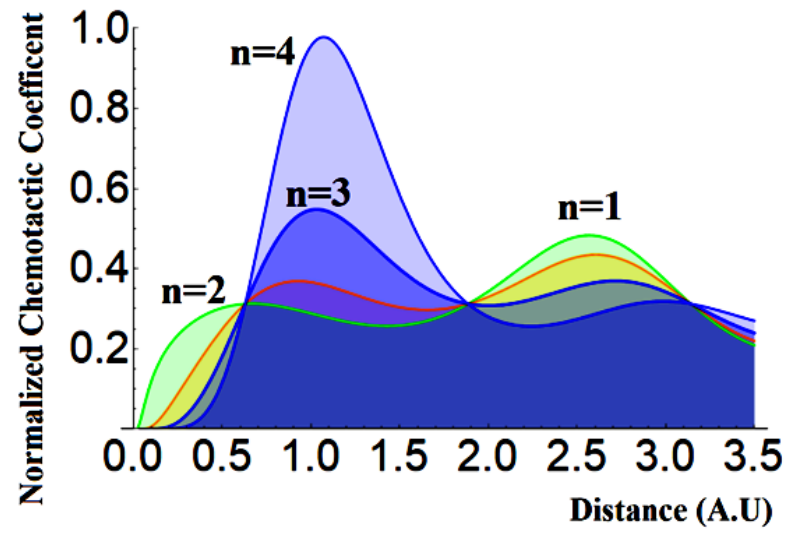

Fig. 1 - The normalized chemotactic coefficient from Eq. 11 for up to 4 values of number " $n$ ", denoting the order of Bessel functions. For this it is assumed the unidimensional representation of $s(x)=\operatorname{Cos}[2.5 x] \operatorname{Sin}\left[0.1 * x^{2}\right]$.

\subsection{Charged Bacteria Density}

In Fig. 1 up to 4 different distributions of the normalized chemotactic coefficient are displayed under the assumption that the substrate density has the quasi-stochastic form given by $\operatorname{Cos}(2.5 x) \operatorname{Sin}\left(0.1 x^{2}\right) \quad$ [10]. This mathematical approach comes from the fact that the existence of periodical manifestation of electric behavior would exhibit both attraction and repulsion, depending the state of pair: bacteriasubstrate.

Thus, positive and negative values with rapid oscillation along the distances are expected to be allowed by the motility of bacteria. One can see that the higher order exhibits a well-shaped peak.

\subsection{The Bessel-Keller-Segel (BKS) Equation}

Therefore from Eq. 5 to Eq. 9 one arrives at a novel version of the Keller-Segel equation called BKS (Bessel-Keller-Segel), that is written below as:

$$
x^{2}\left[\frac{d^{2} b(x, t)}{d x^{2}}\right]+x\left[\frac{d b(x, t)}{d x}\right]+\left(1-\frac{n^{2}}{x^{2}}\right) b(x, t)=0
$$

that is solvable for the bacteria density yielding imminently the integer-order Bessel functions:

$$
b(x, t)=B(x, t)=J_{n}(x, t)
$$

Although a solid interpretation of Eq. 13 might not be adjusted to the scope of this paper, it is possible to some extent to adjudicate a meaning in terms of bacteria motility properties such as cooperative behavior. It is noteworthy that the negative values of Bessel functions are not describing physical solutions. Because of this, the positive values of bacteria density might be interpreted in terms of cooperative and competitive population, depending on the width of distribution. Clearly this requires the option of square of the Bessel functions that provides only physical meaning. Therefore from Eq. 13 one arrives a the solutions given by:

$$
B(x, t) \propto\left|J_{n}(x, t)\right|^{2}
$$

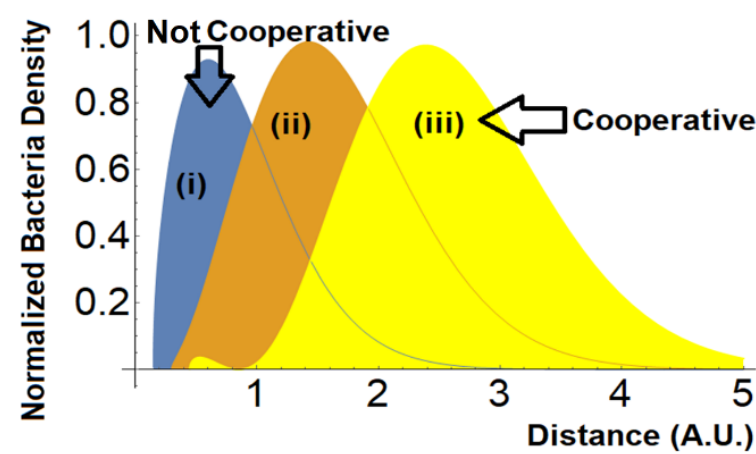

Fig. 2 - The normalized bacteria density $\boldsymbol{B}(\boldsymbol{x}, \boldsymbol{t})$ as a function of distance (in arbitrary units) for the first 3 orders of Bessel functions and their tentative interpretation in terms of cooperative population.

In Fig. 2 up to three possible bacteria density are displayed. For this a normalization function was opted, and it reads $2.9 \lambda_{B} \sqrt{x-n \times \lambda_{A}}$ where $\lambda_{A}$ is about the $5 \%$ of $\lambda_{B}$ for this exercise (this is assumed both free parameters $\lambda_{A}$ and $\lambda_{B}$, to illustrate Fig. 2). Clealrly, both can be changed in more analytic scenarios. Therefore the density spectra for s fixed time " $\boldsymbol{T}$ " reads:

$$
\boldsymbol{B}(\boldsymbol{x}, \boldsymbol{T})=\lambda_{B} \sqrt{x-n \times \lambda_{A}}\left|J_{n}\left(x-n \times \lambda_{A}, \boldsymbol{t}, \boldsymbol{T}\right)\right|^{2}
$$

In fact, inspired at the criterion of [3] in which the bacteria densities have a steady-state profile centered in $x-n \times \lambda_{A}$ being this dependent on the integer " $n$ ". One can see that while the order of Bessel function increases then one would expect the cooperative population moves with distance; as seen in Fig. 2 the distributions moves to the right.

A compelling argumentation of why one calls cooperative population to the distribution (yellow color), can be directly seen in the width of each curve. Thus, when it is larger than the others, one arrives to the fact that bacteria join each other to perform a subsequent action ("Many make force"). A short width is perceived as The number of individuals along the time of interaction can be obtained from a simple integration $\boldsymbol{N}(\boldsymbol{t})=\int \boldsymbol{d} \boldsymbol{x} \boldsymbol{B}(\boldsymbol{x}, t)$. Thus one can 
speculate that from the spatial displacement the velocity can be dependent on the Bessel order. In this view, from Eq. 13 with the time derivative then one arrives at:

$$
\frac{d b(x, t)}{d x} \frac{d x}{d t}=v \frac{d b(x, t)}{d x}=\frac{d J_{n}(x, t)}{d x}
$$

and the straighforward usage of the recurrence relation of the integer-order Bessel function, then one gets

$$
\frac{d b(x, t)}{d x} \frac{d x}{d t} \Rightarrow \frac{d b(x, t)}{d x}=\frac{1}{v}\left[J_{n-1}(x)-J_{n+1}(x)\right] .
$$

Although not any direct interpretation can be extracted from Eq. 17, it is emphasized that only experimental data can provide a robust interpretation and matching if any, from the implementation of Bessel functions in the KellerSegel scenario. Fig. 2 also exhibits a possible competitivity among bacteria along their displacements when all of them are under action of chemotaxis. This displacement per unit of time constitutes the aggregation bacteria velocity " $v$ " as written in right-side of Eq. 17. A tentative explanation about the origin of motility in certain types of bacteria, would be inside the territory of electricity where physics laws could explain inherent properties that are driven by electric forces, either attraction or repulsion. Some studies in the past about that have been reported, for example the one given in [11]. Under this view, the component ionic of bacteria emerges as the main point to board the problem of motility and chemotaxis. With this property, classic electrodynamics emrges as a sustained scenario to go through the bacteria motility properties.

Therefore, one can anticipate that the displacements done by aggregations of bacteria is dictated by classic physics and its corresponding electrodynamics. It is actually not valid for distances less than $0.001 \mathrm{~nm}$ where quantum mechanics governs.

\section{ELECTRODYNAMICS ANALYSIS OF BESSEL-KELLER-SEGEL EQUATION}

A first view to be debated is about the BKS equation's solutions as done in Eq. 13 where the integer-order Bessel functions appear as the possible solutions of bacteria density. One can wonder if the volume integration returns the total electric charge. Actually, this fits well in electrodynamics in the sense that the total charge enclosed in a spherical volumen of radius " $\mathrm{R}$ " (for example, another spatial geometry can be opted as well). A straightforward integration:
$Q(t)=\int_{0}^{R} d V b(x, t)=\int_{0}^{R} 4 \pi r^{2} J_{n}(x, t) d r$ Is seen as the net charge. Below in Fig. 3 the different normalized total charge distributions as a function of sphere radius " $\boldsymbol{R}$ " are displayed. One can see that the ampitude of distributions increases with the radius. For large radius, then one would expect strong attractions as well as repulsion forces, having direct implications with the highest and lowest levels in both cooperativity and competitivity.

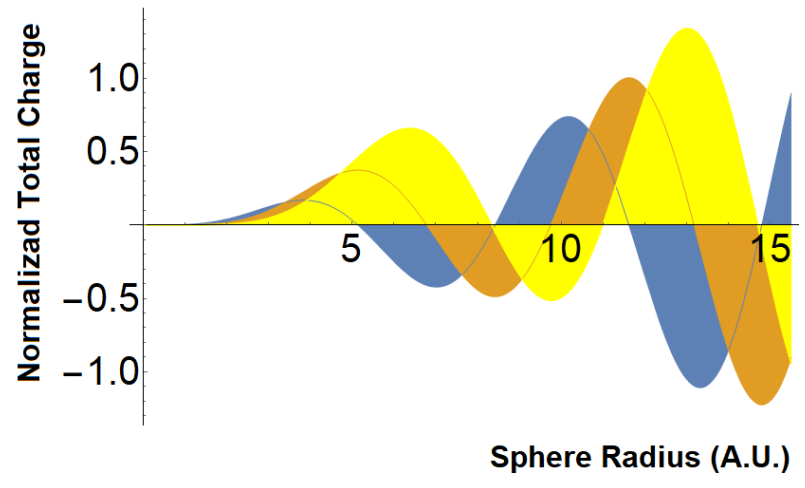

Fig. 3 - The normalized total charge of bacteria against the sphere radius exhibiting oscillations due to the Bessel-like behavior.

Thus in those scenarios of high electric charge for example, one can consider the role of electrodynamics in relation to the level of disruption among populations from the fact that large accumulation of ions would generate electric forces among them. It should be noted that electric interactions can be a reason to disturb the purpose of colonization. This might affect the "so-called cheaters" and other types of social manifestations of bacteria when carrying out actions of chemotaxis. Furthermore, engineered nanodevices recovered of ionic material would constitute an interesting window to attack the problem of infection and the respective diseases caused by bacteria (or virus).

With this background, it is feasible to derive a theory of classical electrodynamics from a BKS equation as expressed in Eq. 12. Thus, it can be written below in conjunction with time evolution as:

$$
\frac{\partial b(x, t)}{\partial t}=x^{2}\left[\frac{d^{2} b(x, t)}{d x^{2}}\right]+x\left[\frac{d b(x, t)}{d x}\right]+\left(1-\frac{n^{2}}{x^{2}}\right) b(x, t)
$$

As seen in previous works, bacteria can transport electrically charged proteins and any type of ions that microorganisms can bring on them. This constitutes an argument to analyze the Keller-Segel equation in terms of electric charge. 
Mathematically speaking, one can integrate the volume in both sides of Eq. 18, so that one gets:

$$
\begin{aligned}
\frac{d}{d t} \int \boldsymbol{b}(\boldsymbol{x}, \boldsymbol{t}) d \boldsymbol{V} & =x^{2} \frac{d^{2}}{d x^{2}} \int \boldsymbol{b}(x, t) d V+x \frac{\boldsymbol{d}}{\boldsymbol{d x}} \int \boldsymbol{b}(\boldsymbol{x}, \boldsymbol{t}) d \boldsymbol{V} \\
& -\left(\mathbf{1}-\frac{\boldsymbol{n}^{2}}{\boldsymbol{x}^{2}}\right) \int \boldsymbol{b}(x, t) d V
\end{aligned}
$$

From the side of electricity, $\boldsymbol{b}(\boldsymbol{x}, \boldsymbol{t})$ denotes the volumetric charge density transported by bacteria, fact that implies that its integration over the volume containing the charge turns out to be the net charge: $\boldsymbol{Q}(\boldsymbol{t})=\int \boldsymbol{d} \boldsymbol{V} \boldsymbol{b}(\boldsymbol{x}, \boldsymbol{t})$. Under this view the left side of Eq. 19 is recognized as the instantaneous electric current. Thus, one arrives at:

$$
\begin{aligned}
\frac{\boldsymbol{d} \boldsymbol{Q}(\boldsymbol{t})}{\boldsymbol{d} \boldsymbol{t}=\boldsymbol{I}(\boldsymbol{t})} & =x^{2} \frac{d^{2}}{d x^{2}} \int \boldsymbol{b}(x, t) \boldsymbol{d} \boldsymbol{V}+\boldsymbol{x} \frac{\boldsymbol{d}}{\boldsymbol{d} \boldsymbol{x}} \int \boldsymbol{b}(x, t) \boldsymbol{d} \boldsymbol{V} \\
& -\left(1-\frac{n^{2}}{x^{2}}\right) \int \boldsymbol{b}(x, t) \boldsymbol{d} \boldsymbol{V}
\end{aligned}
$$

By assuming the cylindrical coordinates system then the variable " $x$ " is now changed to be the radial coordinate. Then one gets:

$$
\begin{aligned}
I(t)= & r^{2} \frac{d^{2}}{d r^{2}} \int b(r, t) d V+r \frac{d}{d r} \int b(r, t) d V \\
& -\left(1-\frac{n^{2}}{r^{2}}\right) Q(t) .
\end{aligned}
$$

Now special attention is paid on the first term of the right side. Since $\boldsymbol{b}(x, t)$ have been defined as the density of charge transported by bacteria, then this term is processed as follows:

$$
r^{2} \frac{d^{2}}{d r^{2}} \int \frac{Q(t)}{A r} d V=\frac{r^{2}}{A} \nabla \int \nabla\left(\frac{Q(t)}{r}\right) d V
$$

where the volume has been assumed to be $\boldsymbol{V}=\boldsymbol{r} \boldsymbol{A}$. The radial derivatives are actually the gradient operator that acts onto the electric potential [12] given by:

$$
\Phi(r)=\frac{Q(t)}{4 \pi \varepsilon r} .
$$

With the balance of units and the incorporation of permissivity constant one arrives at:

$$
\begin{gathered}
r^{2} \frac{d^{2}}{d r^{2}} \int \frac{Q(t)}{A r} d V=\frac{4 \pi \varepsilon r^{2}}{A} \nabla \int \nabla\left(\frac{Q(t)}{4 \pi \varepsilon r}\right) d V \\
=\frac{4 \pi \varepsilon r^{2}}{A} \nabla \int \nabla \Phi(\mathrm{r}) d V .
\end{gathered}
$$

By which one can identify that in the last equation one has the field electric, thus one arrives at the divergence theorem, yielding that this contribution to the BKS equation is proportional to the square of cylindric radius. With this one gets that:

$$
\begin{gathered}
\frac{4 \pi \varepsilon r^{2}}{A} \nabla \int \nabla \Phi(r) d V=\frac{-4 \pi \varepsilon r^{2}}{A} \int \nabla . E d V \\
=\frac{-4 q(t) \pi \varepsilon r^{2}}{A e}
\end{gathered}
$$

Where Gauss's law was employed but in the form of:

$$
\int \nabla . E d V=\frac{q(t)}{e}
$$

With " $e$ " a permissivity constant different to " $\varepsilon$ " because equipotential lines might not be inside the region of electric field in some points of space of bacteria displacement, so that only a single medium is not expected. Instead up two media or more can coexist together. A similar procedure can be applied to the second term of the right side of Eq. 21:

$$
r \frac{d}{d r} \int b(x, t) d V=\frac{4 \pi \varepsilon r}{A} \int \nabla\left(\frac{Q(t)}{4 \pi \varepsilon r}\right) d V
$$

and again, one can construct the electric potential yielding finally that the term of Eq. 27 is linearly proportional to the cylindric radius:

$$
\begin{gathered}
\frac{4 \pi \varepsilon r}{A} \int \nabla\left(\frac{Q(t)}{4 \pi \varepsilon r}\right) d V= \\
\frac{4 \pi \varepsilon r}{A} \int \nabla \Phi(\mathrm{r}) d V=\frac{-4 \pi \varepsilon r L}{A} \int \mathrm{E} d A=\frac{-4 q(t) \pi \varepsilon r L}{A e} .
\end{gathered}
$$

It should be noted again that $\boldsymbol{d} \boldsymbol{V}=\boldsymbol{L} \boldsymbol{d} \boldsymbol{A}$. Therefore, when previous resulting equations done above are inserted in Eq. 21 then one arrives at:

$$
\begin{aligned}
\frac{d \mathcal{Q}(t)}{d t} & =\left[-\frac{4 q(t) \pi \varepsilon r^{2}}{A e}-\frac{4 q(t) \pi \varepsilon r L}{A e}\right. \\
& \left.-\left(1-\frac{n^{2}}{r^{2}}\right)\right] \mathcal{Q}(t) .
\end{aligned}
$$

Thus, one can see that all charges of the right side have a negative sign. Putting apart the total charge, then one arrives at:

$$
\frac{d Q(t)}{d t}=\mathcal{Q}(t)\left[-\frac{4 \pi \varepsilon r^{2}}{A e}-\frac{4 \pi \varepsilon r L}{A e}-\left(1-\frac{n^{2}}{r^{2}}\right)\right] .
$$

One can see that the apparition of sign "-" on the right side is because the possible existence of a type of discharge because the straightforward solution of Eq. 30 yields the familiar negative exponential.

Electric interactions between compounds can be measured through the electric force as dictated by classical electrodynamics. To explore this, the total charge of bacteria should be explicitly done, thus from Eq. 30,

$$
\frac{d Q(t)}{\mathcal{Q}(t)}=\left[-\frac{4 \pi \varepsilon r^{2}}{A e}-\frac{4 \pi \varepsilon r L}{A e}-\left(1-\frac{n^{2}}{r^{2}}\right)\right] d t,
$$


whose integration yields the closed-form solution:

$$
\boldsymbol{Q}(\boldsymbol{t})=\boldsymbol{Q}_{0} \operatorname{Exp}\left[-\int_{0}^{T} \frac{4 \pi \varepsilon}{\boldsymbol{A e}}\left(\boldsymbol{r}^{2}+\boldsymbol{r}+\boldsymbol{\eta}\left[1-\frac{n^{2}}{\boldsymbol{r}^{2}}\right]\right) \boldsymbol{d t}\right]
$$

With $\boldsymbol{\eta}=\frac{A e}{4 \pi \varepsilon}$ and by specifying the upper limit by "T" the net charge that is transported by the bacteria with the change $\boldsymbol{R}(\boldsymbol{r})=\boldsymbol{r}^{2}+\boldsymbol{r}+\boldsymbol{\eta}\left[1-\frac{n^{2}}{\boldsymbol{r}^{2}}\right]$, then Eq. 32 can be written in compact form as:

$$
\mathcal{Q}(T, r)=Q_{0} \operatorname{Exp}\left[-\frac{4 \pi \varepsilon T}{A e} R(r)\right] .
$$

With this result, now the electric current that involves a more general electric description of bacteria aggregation transporting ions is given by the instantaneous derivative of Eq. 33 and written as:

$I(t=T, r)=-\frac{4 Q_{0} \pi \varepsilon T}{A e} \frac{d R(r, t)}{d t} \operatorname{Exp}\left[-\frac{4 \pi \varepsilon T}{A e} R(r)\right]$.

On the other hand in the simplest case by which " $r$ " does not depend on time, then the integration over the time variable inside the exponential in Eq. 32 is trivial. In this manner the resulting electric current can be written in a simplified form as:

$$
\frac{d Q(t)}{d t}=I(T)=-\frac{4 Q_{0} \pi \varepsilon T}{A e} R(r) \operatorname{Exp}\left[-\frac{4 \pi \varepsilon T}{A e} R(r)\right] .
$$

Actually the variable " $T$ " can be understood as the period in the which the bacteria aggregation behaves as an electric current.

\subsection{Biophysics Interpretation of Electrical Currents}

Eq. 34 is displayed in Fig. 4 exhibiting a minimum for $r \approx 2$ a.u. For this plotting it was used as a numerical expression for Eq. 34 in the form of:

$$
\begin{array}{r}
I(t=T, r)=0.0001\left(2 \mathrm{r}+0.01+\frac{8}{(\mathrm{r})^{\wedge} 3}\right) \operatorname{Exp}\left[-0.01\left(\mathrm{r}^{\wedge} 2+\right.\right. \\
\left.\left.0.01 \mathrm{r}+\left(1-\frac{4}{(\mathrm{r})^{\wedge}}\right)\right)\right] .
\end{array}
$$

As indicated at Fig. 4, bacteria aggregation would exhibit a kind of disruption as seen in the minimum value of current distribution. In this manner, one can wonder if it is an inherent property of bacteria aggregation or if it is a pure speculative theoretical result that might not be matched with experiments. It should be noted that all these procedures have been done under the assumption of a 1-Dimension model. Of course, realistic simulations might be necessary in order to identify rupture of electrical properties. As done in [14], memory-based chemotaxis would exibit drift velocities. So that one can argue that this drift dynamics might appear from electric phenomena more than a pure biological reason.

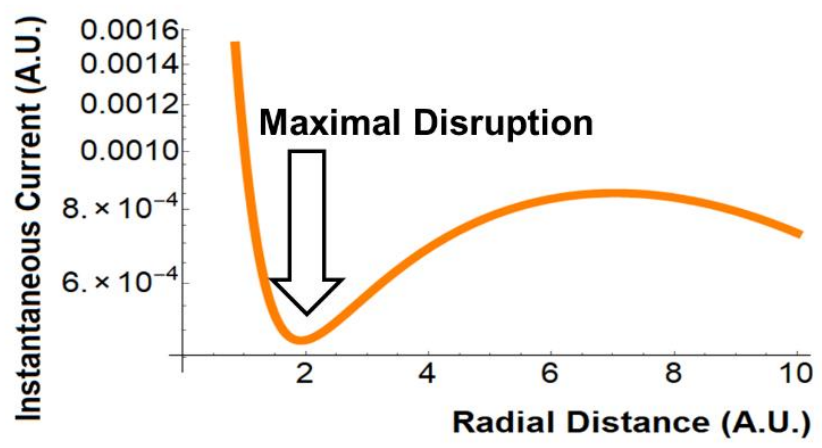

Fig. 4 - The instantaneous current as a function of radial distance (Eq.36), expressed in in arbitrary units. Two phases can be perceived.

Below in Fig. 5 the simplest scenario of Eq. 35 written as $I(t)=t \operatorname{Exp}(-t)$ is illustrated. The qualitative shape of electric current indicates its maximal value. Clearly, it is directly interpreted as the inverse scenario of Fig. 4 establishing a kind of complementarity with it. This triggers logic scenario establishing that organization is first and disruption is after.

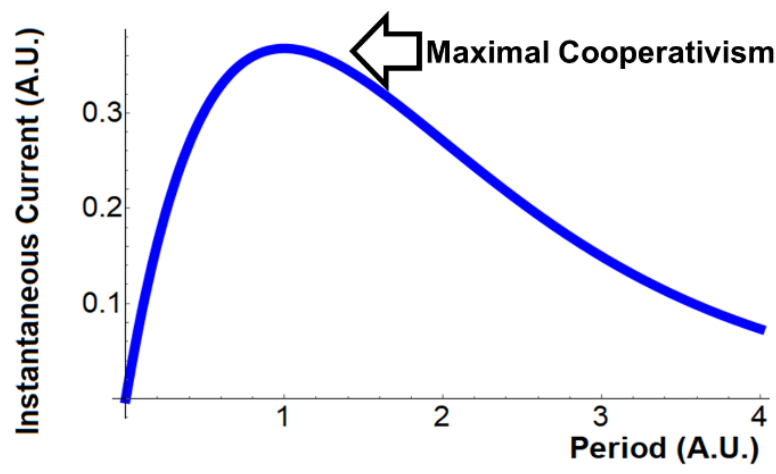

Fig. 5 - RC Discharge: The instantaneous current and period of electric interaction of bacteria as a function of radial distance, both expressed in in arbitrary units. This plot as well as Fig. 1, Fig. 2, Fig. 3 and Fig. 4 were done with the usage of Wolfram [13]

In this manner, one can see that from Fig. 4 and Fig. 5 the possible existence of well-defined phases. This would characterize the BKS model. These possible phases would emerge from the fact that the Eq. 5 exhibits a kind of electric discharge as a RC-circuit. It is in accordance to the negative exponential of Eq. 35 . Therefore, bacteria aggregation and their social manifestations would be disrupted. In Fig. 5 the instantaneous current falls down as a fact that bacteria have "finshed" a social action leaving them to break down the possible molecular communications between them. 


\section{CONCLUSION}

In this paper, an extension of the Keller-Segel equation has been established. It has taken advantage of having a second-order differential equation to establish a novel Bessel-Keller-Segel equation by which, at a first instance, it would give information about the electric behavior of bacteria and the possible implications of this in the social behavior. This appears to be crucial in prospective nanonetworks. Therefore, the electrodynamics of an electrically charged bacteria aggregation has been derived through closed-form equations. Interestingly, the shape of the curve of derived current exhibits a kind of discharge. Although a tentative interpretation in terms of social disruption because social behavior of the bacteria population is assumed, simulations and experimental studies should be done to corroborate the theory of this paper. The assumptions made throughout this paper have served to minimize the mathematical load that involves a second-order differential equation. Indeed the resulting curves have kept a close relationship with well-known electrodynamics.

In future work, some well-known families of bacteria and their data will be employed to explore the possible similarities with the present proposal.

\section{REFERENCES}

[1] E. F. Keller and L. A. Segel, Model for chemotaxis, J. Theor. Biol. 30 (1971) 225234.

[2] Michael Vilkhovoy, Mason Minot and Jeffrey D. Varner, Effective Dynamic Models of Metabolic Networks, IEEE Life Sciences Letters, Volume: 2, Issue: 4, 2016.

[3] Bige D. Unluturk, Sasitharan, Balasubramaniam, Ian F. Akyildiz, The Impact of Social Behavior on the Attenuation and Delay of Bacterial Nanonetworks, IEEE Transactions on NanoBioscience, Year: 2016 Volume: 15, Issue: 8.

[4] I. F. Akyildiz, The internet of Bio-Nano things, IEEE Communications Magazine Year: 2015, Volume: 53, Issue: 3, Pages: 32 - 40.
[5] X. Li, Y. Wang, Z. Xiang. Global existence and boundedness in a 2D Keller-Segel-Stokes system with nonlinear diffusion and rotational flux. Commun. Math. Sci., 14 (2016).

[6] Gerald Rosen, Navier-Stokes symmetry in the phenomenological transport theory for bacterial chemotaxis, Phys, Rev. A 29, 5, May 1984.

[7] Estrada-Rodriguez, Gissell, Gimperlein, HeikoPainter, Kevin. (2017). Fractional Patlak-Keller--Segel Equations for Chemotactic Superdiffusion. SIAM Journal on Applied Mathematics. 78. 10.1137/17M1142867.

[8] T. Xiang, "How strong a logistic damping can prevent blow-up for the minimal KellerSegel chemotaxis system?," J. Math. Anal. Appl. 459, 1172-1200 (2018). https://doi.org/10.1016/j.jmaa.2017.11.022.

[9] Fournier, N., \& Jourdain, B. (2015). Stochastic particle approximation of the Keller-Segel equation and two-dimensional generalization of Bessel processes. Annals of Applied Probability, 27, 2807-2861.

[10] T. Black. Sublinear signal production in a twodimensional Keller-Segel-Stokes system Nonlinear Anal. Real World Appl., 31 (2016), pp. 593-609.

[11] Huber Nieto-Chaupis, Macrophage-Like Nanorobots To Anticipate Bacterial Dynamics, 2019 IEEE 9th Annual Computing and Communication Workshop and Conference (CCWC) Year: 2019, Conference Paper.

[12] J. D. Jackson, Classical Electrodynamics, 3rd Edition, Chap-I BoundaryValue Problems in Electrostatics: I. Wiley 1998.

[13] Wolfram Mathematica, www.wolfram.com.

[14] Gosztolai, A., Barahona, M. Cellular memory enhances bacterial chemotactic navigation in rugged environments. Commun Phys 3,47 (2020). https://doi.org/10.1038/s42005020-0312-8. 


\section{AUTHOR}

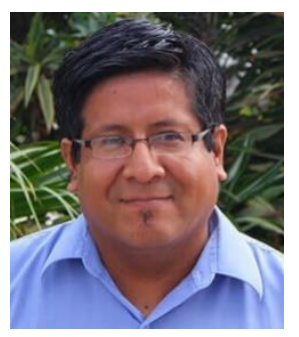

Huber Nieto-Chaupis holds a Bachelor in Physics (1996) from the Universidad Nacional Mayor de San Marcos in Lima,Perú, a Licenciado in Physics (1999) from Universidad Nacional Mayor de San Marcos, a Master in Physics from Pontificia Univrsidad Católica del Perú (2003) and a Doktor in Rerum Naturalium Physik Experimental from Humboldt Universität in Berlin, Germany (2007). He has been a researcher at the Academia Sinica in Taiwan, Province of China (2007) and the University of Science and Technology of China in Hefeim, People's Republic of China (2009). In Perú, he has worked at the National University of Engineering UNI (2008-2011) and from 2011 to 2018 was an independent researcher working in various Peruvian private universities. In 2019, he joined the Universidad Autónoma del Perú as a senior principal investigator. $\mathrm{He}$ is interested in Nanomedicine, Internet of Bio-Nano Things, Photon science, Quantum Mechanics and Machine Learning. 